\title{
A Hand Prosthesis with an Under-Actuated and Self-Adaptive Finger Mechanism
}

\author{
R. A. R. C. Gopura*, D. S. V. Bandara \\ Bionics Laboratory, Department of Mechanical Engineering, University of Moratuwa, Katubedda, Sri Lanka \\ Email: *gopurar@mech.mrt.ac.lk
}

How to cite this paper: Gopura, R.A.R.C. and. Bandara, D.S.V (2018) A Hand Prosthesis with an Under-Actuated and Self-Adaptive Finger Mechanism. Engineering, 10, 448-463. https://doi.org/10.4236/eng.2018.107031

Received: June 19, 2018

Accepted: July 20, 2018

Published: July 23, 2018

Copyright $\odot 2018$ by authors and Scientific Research Publishing Inc. This work is licensed under the Creative Commons Attribution International License (CC BY 4.0).

http://creativecommons.org/licenses/by/4.0/

(c) (i) Open Access

\begin{abstract}
One of the major problems faced by hand amputees is the unavailability of a lightweight and powered multi-functional hand prosthesis. Under-actuated finger designs play a key role to make the hand prosthesis lightweight. In this paper, a hand prosthesis with an under-actuated and self-adaptive finger mechanism is proposed. The proposed finger is capable to generate passively different flexion/extension angles for a proximal interphalangeal (PIP) joint and a distal interphalangeal (DIP) joint for each flexion angle of metacarpophalangeal (MCP) joint. In addition, DIP joint is capable to generate different angles for the same angle of PIP joint. Hand prosthesis is built on the proposed finger mechanism. The hand prosthesis enables user to grasp objects with various geometries by performing five grasping patterns. Thumb of the hand prosthesis includes opposition/apposition in addition to flexion/extension of MCP and interphalangeal (IP) joint. Kinematic analysis of the proposed finger has been carried out to verify the movable range of the joints. Simulations and experiments are carried out to verify the effectiveness of the proposed finger mechanism and the hand prosthesis.
\end{abstract}

\section{Keywords}

Under Actuation, Self-Adaptation, Four-Bar Mechanism, Finger Mechanism, Hand Pros-Thesis

\section{Introduction}

Hand prosthesis is an artificial device which replaces the missing hand of an amputee and is expected to fulfil the functional and aesthetic requirements of the amputated hand. The main problem that causes amputees not to accept the available prostheses is the unavailability of light-weight prostheses with acceptable controlling and functional properties [1]. It has been identified that the 
thumb and the index finger play an important role than the other fingers in most of daily grasping activities [2]. Therefore, many hand prostheses are based on three fingered configurations and finger abduction/adduction is not considered in the designs [3]. During activities of daily living (ADL), hand is arranged in different grasping patterns to handle objects with different geometries [4]. Metacarpophalangeal (MCP), Proximal Interphalangeal (PIP) and Distal Interphalangeal (DIP) are manipulated to obtain different configurations to generate different grasping patterns. Flexion/extension angles of these joints are arranged in different combinations to realize different grasping patterns. Subsequently, a hand prosthesis should also be developed with the ability of generating different grasping patterns to help the amputee during ADL. Studies presented in [5] [6], and [7] attempt to accommodate these requirements in their devices. However, very few demonstrate the ability to provide human alike motion in their mechanisms. Generally, a robotic hand prosthesis should be lightweight while providing required dexterity and functionality. In order to arrive at a suitable compromise, researchers are trying to reduce number of actuators while trying to achieve maximum functionality of the prosthesis. In view of that, under-actuated mechanisms have been developed and tested. When a mechanism has lesser number of actuators than the generated degrees of freedom (DOF) [8] the mechanism is said to be under-actuated. Several researchers [3] [9]-[16] [19]-[24] have proposed under-actuated finger mechanisms and hand designs. Most of the fingers are capable of generating human alike cylindrical grasping mode to grasp cylindrical objects. The Smart Hand [7] is a transradial prosthesis with under-actuated fingers. The finger mechanism proposed in [15] is capable of passively generating different angles for PIP and DIP joints for each flexion angle of MCP joint. The angles change according to the grasping object passively and the thumb has a single DOF. However, DIP joint is not capable of generating different angles for the same angle of PIP joint. Therefore, if an object directly touches with the middle phalange while grasping the self-adaptation ability of DIP joint is lost.

Therefore, in this research a prosthetic hand comprises of under-actuated and self-adaptive fingers is proposed. The proposed finger is used as the index, middle, ring or little finger of the hand prosthesis. Modified mechanism of the proposed finger is used as thumb of the hand prosthesis which includes thumb opposition/apposition in addition to flexion/extension of MCP joint and interphalangeal (IP) joint. The proposed finger is capable of passively generating different flexion/extension angles for a PIP joint and a DIP joint for each flexion angle of MCP joint. In addition, DIP joint is capable of generating different angles for the same angle of PIP joint. In this study, abduction/adduction of MCP joint is not considered and only the flexion/extension is considered. The hand prosthesis assists user to grasp objects with various geometries by performing cylindrical grasp, hook grasp, lateral pinch and tip pinch and palmar pinch.

Next section of the paper proposes the under-actuated and self-adaptive finger 
mechanism. Section 3 presents the mechanical design and the mechanisms of the introduced hand prosthesis. Experiments and results are presented in section 4 and the last section concludes the paper.

\section{Under-Actuated and Self-Adaptive Finger}

Proposed finger can generate flexion/extension of MCP, PIP and DIP joints. It can be used as an index, middle, ring or little fingers of a hand prosthesis. Main structure of the finger can be simplified to a mechanism which consists of two four-bar mechanisms which are combined at PIP joint and with a coupling linkage as shown in Figure 1. Linkage for distal phalanx is connected to the second four-bar mechanism at DIP joint. As shown in Figure 1, three torsion springs are attached between lower bar-1 (proximal phalanx) and palm at MCP joint, lower bar-1 and driving bar at first four-bar mechanism; lower bar-2 (middle phalanx) and the side bar-1 at PIP joint; respectively. Driving bar is coupled to the motor which generates the driving torque. Second torsion spring is used to limit each joint motion of the first four-bar mechanism in its predefined initial position and carrying out the under-actuation. Third torsion spring is used to keep the second four-bar mechanism as a rigid body relative to first four-bar mechanism. The ratio of spring constants of first, second and third springs is 6.4:10:1.

\subsection{Under-Actuation of the Finger}

Initially, when the torque is applied by the driving bar the finger operates as a single rigid body due to second and third torsion springs. Then, first torsion spring which has lower spring constant than second torsion spring starts to compress and the spring resistance increases. When the spring resistance of second torsion spring is overcome by the first torsion spring middle phalanx starts to rotate relatively to the proximal phalanx. Once the middle phalanx motion is restricted by the grasped object, third torsion spring is compressed and side bar-1 starts rotating relative to middle phalanx. Then, distal phalanx

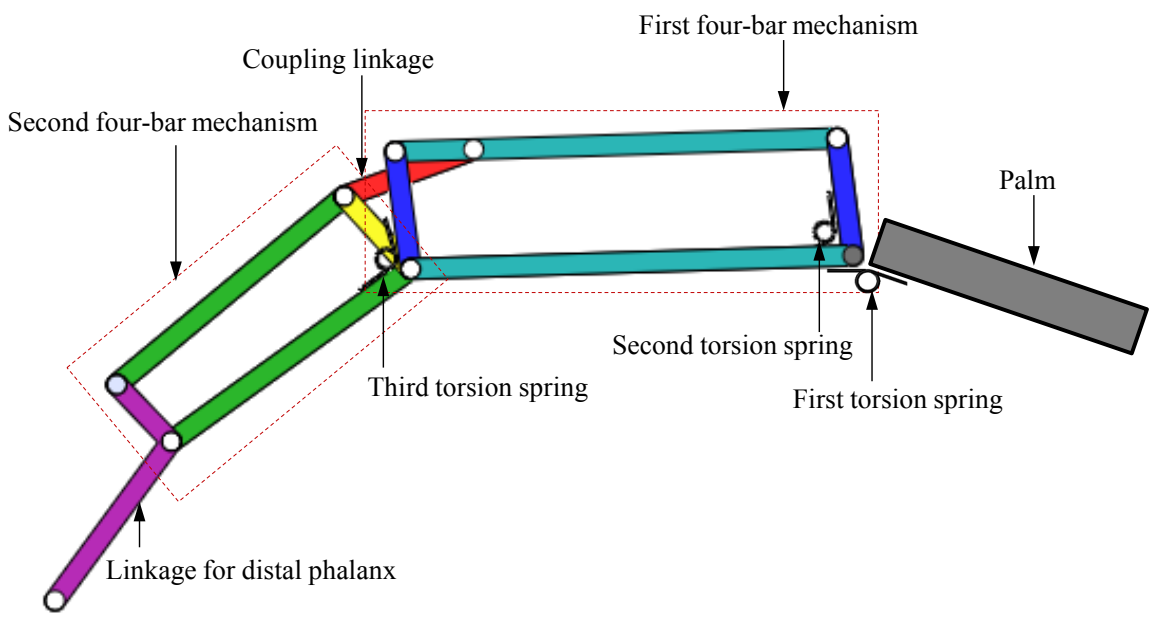

Figure 1. Proposed finger mechanism. 
continues rotating relative to middle phalanx. In order to carry out the under-actuation properly second torsion spring should have the highest spring constant, first torsion spring should have the second highest spring constant and third torsion spring should have the lowest spring constant.

In order to carry out the proper under-actuation;

$$
K_{\text {Third Spring }}<K_{\text {First Spring }}<K_{\text {Second Spring }}
$$

where $K$ is spring constant.

\subsection{Self-Adaptation of the Finger}

Proposed finger incorporates self-adaptation ability which enables to grasp objects with different geometries. Figure 2 demonstrates how the finger achieves self-adaptation according to the shape of the object. When the proximal phalanx motion is restricted due to the geometry of an object as in the Figure 2(a), the driving bar continues to rotate relative to the proximal phalanx, compressing second torsion spring. It rotates the second four-bar linkage until the middle phalanx touches the object as illustrated in the Figure 2(b). Once the middle phalanx motion is restricted by the grasped object, third torsion spring is compressed and side bar-1 starts rotating relative to middle phalanx. Then, distal phalanx continues rotating relative to middle phalanx until the object is grasped properly. Thus, the joint angles show the capability to adopt their values passively according the geometry of the object.

\subsection{The Thumb}

Proposed finger mechanism shown in Figure 1 can be modified and used for the thumb. The main structure of the thumb is shown in Figure 3. The thumb is similar to the proximal phalanx and middle phalanx of the finger in Figure 1. It consists of a four-bar mechanism and two torsional springs. Thumb can generate flexion/extension of its MCP and IP. Additionally, opposition/apposition of thumb can be generated as explain in Section 3 [refer Figure 7(c)]. When the motor torque is applied by the driving bar the thumb operates as a single rigid body due to second spring. Then, lower bar (proximal phalanx) rotates and first torsion spring starts to compress and the spring resistance increases. When the

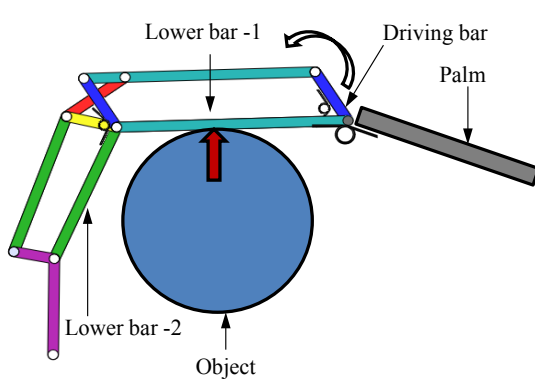

(a)

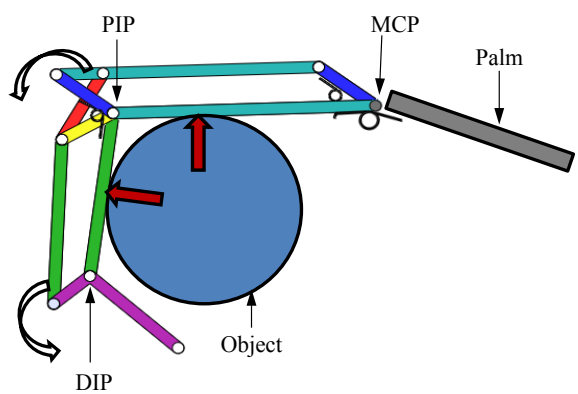

(b)

Figure 2. Self-adaptation mechanism of the finger. 


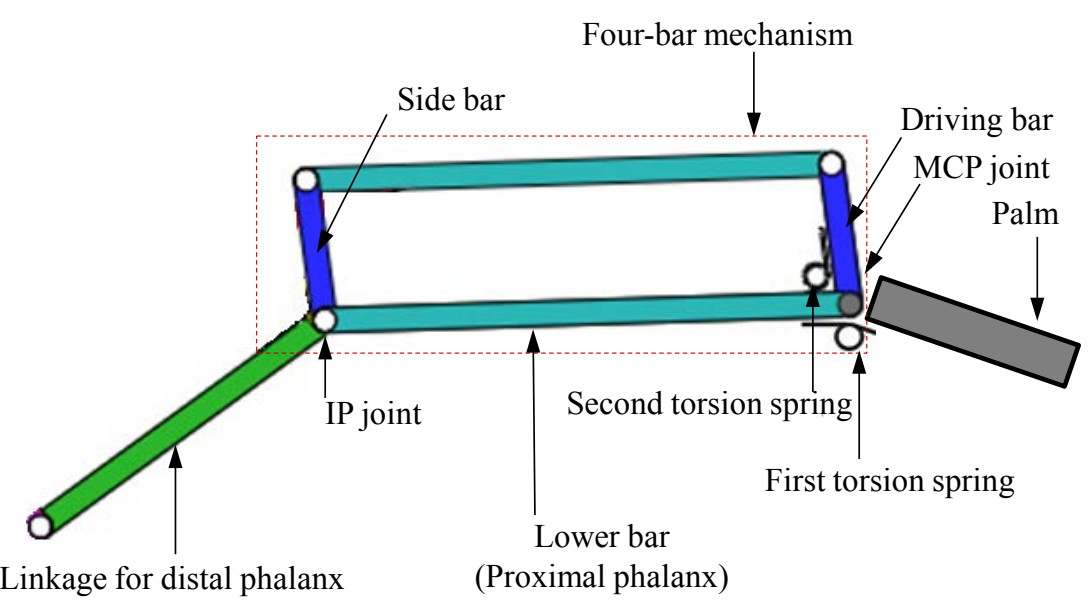

Figure 3. Thumb mechanism.

spring resistance of second torsion spring is overcome by the first torsion spring, distal phalanx starts to rotate relatively to the proximal phalanx. The ratio of spring constants of first and second springs is 0.64:1.

\subsection{Kinematic Analysis of the Finger}

In order to understand the kinematic behaviour of the finger mechanism kinematic analysis is carried out. As shown in Figure 4 and Figure 5 the joints of the fingers are connected with nine mechanical links: $\mathrm{AC}, \mathrm{AB}, \mathrm{CD}, \mathrm{BD}, \mathrm{EF}, \mathrm{BF}, \mathrm{BH}$, FG and GHI. Joint angle relationship between these linkages gives the respective motion between phalanxes of the finger. AC is rigidly connected to the wheel of the worm-and-wheel gear which is driven by the motor to drive the whole mechanism. Therefore, AC can rotate with respect to joint $\mathrm{A}$ when the motor rotates. Rotation of $\mathrm{AC}$ actuates $\mathrm{CD}$. Then due to the compression of the torsion spring between $\mathrm{AB}$ and $\mathrm{AC}, \mathrm{AB}$ is actuated by $\mathrm{AC}$. $\mathrm{EF}$ and $\mathrm{BD}$ both are actuated by $\mathrm{CD}$, and $\mathrm{EF}$ actuates both $\mathrm{BF}$ and FG. When $\mathrm{BD}$ actuates, due to the compression of torsion spring between $\mathrm{BH}$ and $\mathrm{BD}, \mathrm{BH}$ starts to actuate. At the end, the GHI is actuated by FG.

First, second and third springs are connected between, $A B$ and the palm; $A B$ and $\mathrm{AC}$; and $\mathrm{BD}$ and $\mathrm{BH}$. Initially even though the torque is applied to the $\mathrm{AC}$, angle between $\mathrm{AB}$ and $\mathrm{AC}(\theta)$ is kept constant due to the torsion spring between $\mathrm{AB}$ and $\mathrm{AC}$. Then, angular velocity of $\mathrm{AB}$ becomes zero when its motion is restricted by the object. Initially, $\theta$ is known. Thus, $\beta=90-(\alpha+\theta)$ for any $\alpha$ value. Assume that $\mathrm{AC}, \mathrm{AB}, \mathrm{CD}, \mathrm{DB}, \mathrm{EF}, \mathrm{BF}, \mathrm{FG}, \mathrm{BH}, \mathrm{GH}, \mathrm{HI}, \mathrm{DE}$ are $l_{1}$ to $l_{11}$ respectively. Considering $\mathrm{ABDC}$ four-bar mechanism;

$$
\begin{aligned}
& l_{1} \sin \alpha+l_{3} \sin \gamma=l_{2} \cos \beta+l_{4} \sin \delta \\
& l_{1} \cos \alpha+l_{3} \cos \gamma=l_{2} \sin \beta+l_{4} \cos \delta
\end{aligned}
$$

From (2),

$$
\sin ^{2} \delta=\frac{\left(l_{1} \sin \alpha+l_{3} \sin \gamma-l_{2} \cos \beta\right)^{2}}{\left(l_{4}\right)^{2}}
$$




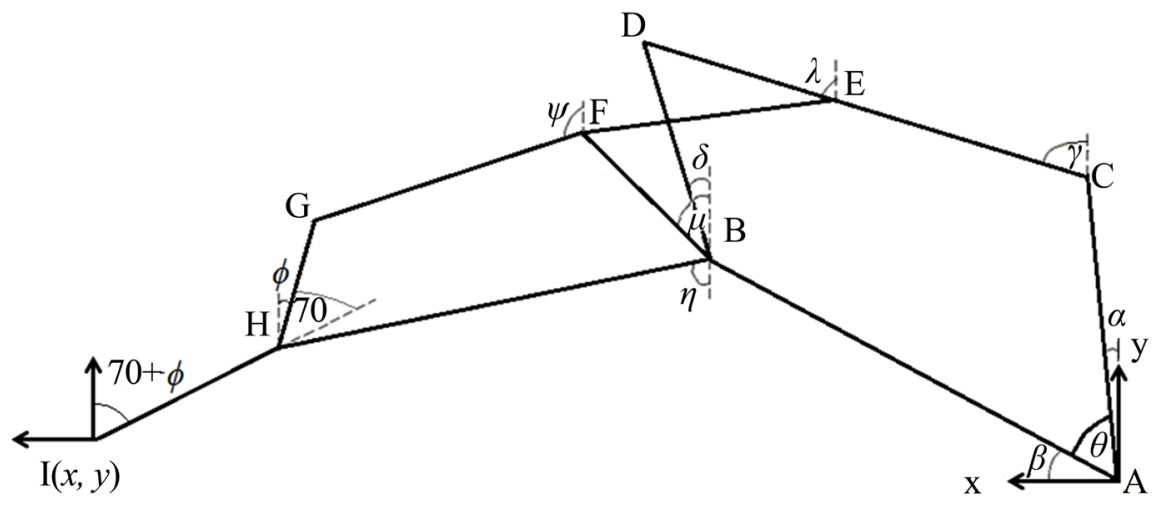

Figure 4. Kinematic diagram of the index finger [25].

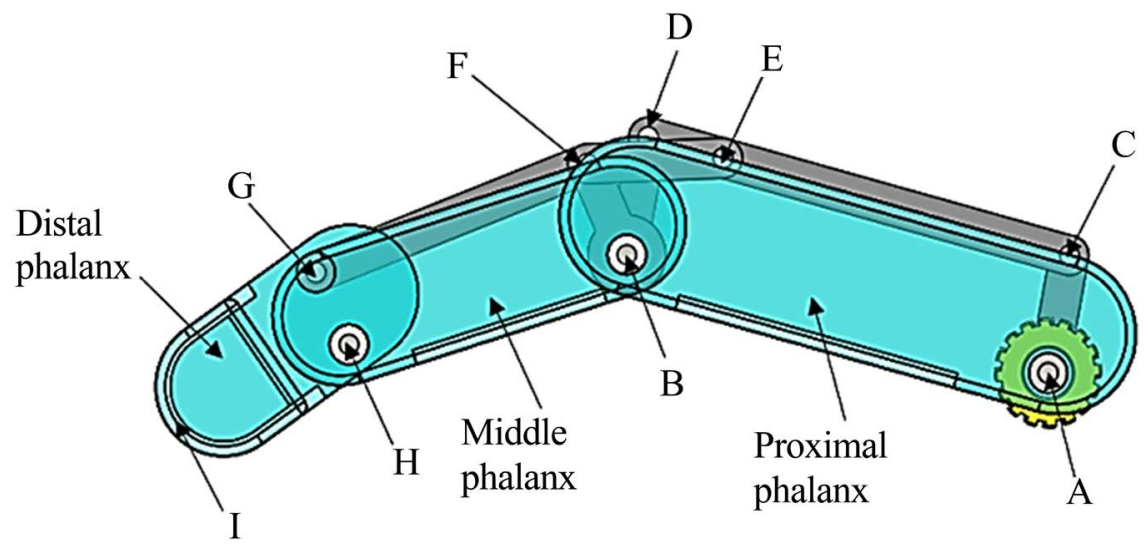

Figure 5. 3D model of the index finger.

From (3),

$$
\cos ^{2} \delta=\frac{\left(l_{1} \cos \alpha+l_{3} \cos \gamma-l_{2} \sin \beta\right)^{2}}{\left(l_{4}\right)^{2}}
$$

$\gamma$ can be found for the given $\alpha$ and $\beta$ from the equation given below.

$$
\begin{aligned}
& l_{1} \cos (\gamma-\alpha)-l_{2} \sin (\gamma+\beta) \\
& =\frac{1}{2\left(l_{3}\right)}\left[\left(l_{4}\right)^{2}-\left(l_{1}\right)^{2}-\left(l_{2}\right)^{2}-\left(l_{3}\right)^{2}+2 l_{1} l_{2} \sin (\alpha+\beta)\right]
\end{aligned}
$$

Similarly, solving (2) and (3),

$$
\begin{aligned}
& l_{2} \sin (\delta+\beta)-l_{1} \cos (\delta-\alpha) \\
& =\frac{1}{2\left(l_{4}\right)}\left[\left(l_{3}\right)^{2}-\left(l_{2}\right)^{2}-\left(l_{1}\right)^{2}-\left(l_{4}\right)^{2}+2 l_{1} l_{2} \sin (\alpha+\beta)\right]
\end{aligned}
$$

The angle $(\delta$ ) can be found from the above equation for the given $\alpha$ and $\beta$. Therefore, $\mu$ can be found using $\delta$ and angle $\gamma$. Consider DE, EF, BF and BD.

$$
\begin{gathered}
l_{11} \cos \gamma-l_{5} \cos \lambda=l_{4} \cos \delta-l_{6} \cos \mu \\
l_{11} \sin \gamma-l_{5} \sin \lambda=l_{4} \sin \delta-l_{6} \sin \mu
\end{gathered}
$$

Solving the above equations, $\lambda$ can be found from the below equation. 


$$
\begin{aligned}
& l_{4} \cos (\lambda-\delta)-l_{11} \cos (\lambda-\gamma) \\
& =\frac{1}{2\left(l_{5}\right)}\left[\left(l_{6}\right)^{2}-\left(l_{4}\right)^{2}-\left(l_{5}\right)^{2}-\left(l_{11}\right)^{2}+2 l_{4} l_{11} \cos (\gamma-\delta)\right]
\end{aligned}
$$

$\mu$ also can be found similarly from the below equation.

$$
\begin{aligned}
& l_{11} \cos (\mu-\gamma)-l_{4} \cos (\mu-\delta) \\
& =\frac{1}{2\left(l_{6}\right)}\left[\left(l_{5}\right)^{2}-\left(l_{4}\right)^{2}-\left(l_{6}\right)^{2}-\left(l_{11}\right)^{2}+2 l_{4} l_{11} \cos (\gamma-\delta)\right]
\end{aligned}
$$

Therefore, $\varphi$ can be found using $\mu$ and $\eta$. Considering BFGH four-bar mechanism;

$$
\begin{aligned}
& l_{8} \sin \eta=l_{6} \sin \mu+l_{7} \sin \varphi+l_{9} \sin \phi \\
& l_{8} \cos \eta=-l_{6} \cos \mu-l_{7} \cos \varphi+l_{9} \cos \phi
\end{aligned}
$$

From (6) and (7), $\varphi$ can be found as below.

$$
\begin{aligned}
& l_{8} \cos (\phi-\eta)+l_{6} \cos (\phi+\mu) \\
& =\frac{1}{2 l_{9}}\left[\left(l_{8}\right)^{2}+\left(l_{6}\right)^{2}+\left(l_{9}\right)^{2}-\left(l_{7}\right)^{2}+2 l_{6} l_{8} \cos (\eta+\mu)\right]
\end{aligned}
$$

Similarly, $\varphi$ can be found from the equation given below.

$$
\begin{aligned}
& l_{8} \cos (\psi+\eta)+l_{6} \cos (\psi-\mu) \\
& =\frac{1}{2 l_{7}}\left[\left(l_{9}\right)^{2}-\left(l_{8}\right)^{2}+\left(l_{6}\right)^{2}-\left(l_{7}\right)^{2}+2 l_{6} l_{8} \cos (\eta+\mu)\right]
\end{aligned}
$$

Considering joints of a finger fingertip " $P$ ", position, $(x, y)$ and orientation, $(70+\varphi)$ with respected to the motor shaft $\mathrm{A}(0,0)$ can be found.

$$
\begin{aligned}
& x=l_{2} \cos \beta+l_{8} \sin \eta+l_{10} \sin (70+\phi) \\
& y=l_{2} \sin \beta-l_{8} \cos \eta+l_{10} \cos (70+\phi)
\end{aligned}
$$

(8) and (9) can be used to derived the position and orientation of the fingertip relative to the palm.

\section{Proposed Hand Prosthesis}

The proposed finger and thumb is used to introduce a multi-functional hand prosthesis shown in Figure 6. Table 1 shows summary of specification of the proposed hand prosthesis.

\section{Mechanical Design and Mechanism}

The hand prosthesis consists of four main units: first finger unit, second finger unit, third finger unit and a palm [refer Figure 6]. Since the index finger and the thumb play an important role than the other fingers in most of daily grasping activities [2] those two are taken as separate finger units and the middle, ring and little fingers together are taken as a separate unit for the actuation. First finger unit consists of the index finger, a motor and worm and wheel gears (reduction ratio 35:1) as shown in Figure 7(a). Second finger unit consists of three 
Table 1. Specification of the proposed hand prosthesis.

\begin{tabular}{cc}
\hline Specifications & Quality \\
\hline Finger length & $95 \mathrm{~mm}$ \\
Palm width & $83 \mathrm{~mm}$ \\
Palm thickness & $25 \mathrm{~mm}$ \\
Flexion range of MCP joint & 0 to 90 Degrees \\
\hline
\end{tabular}

First finger unit

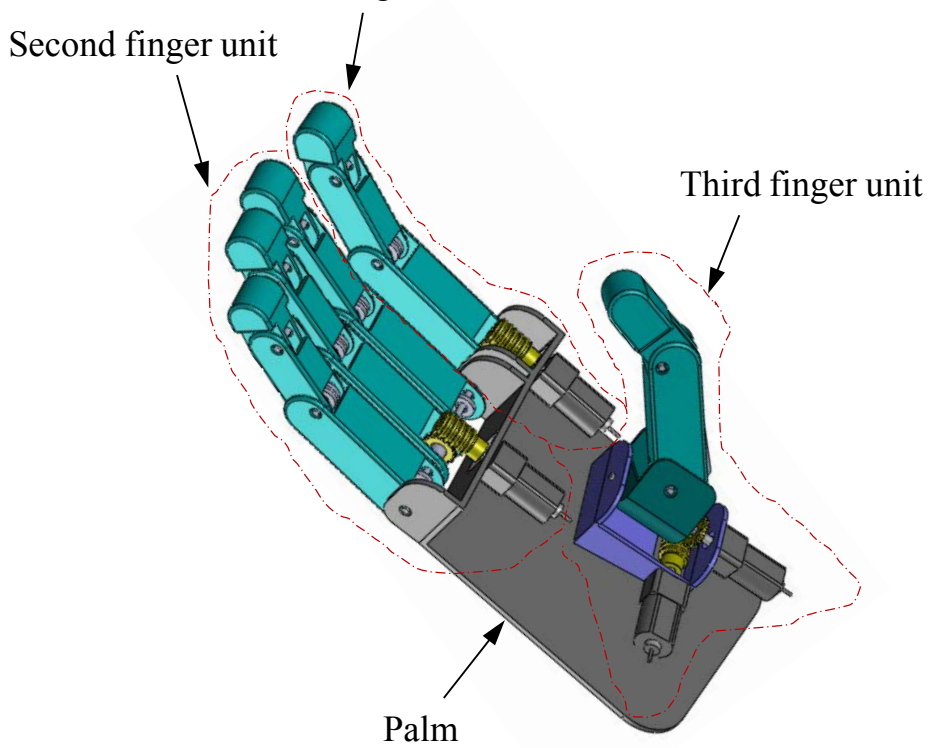

Figure 6. 3D model of Proposed Hand Prosthesis.

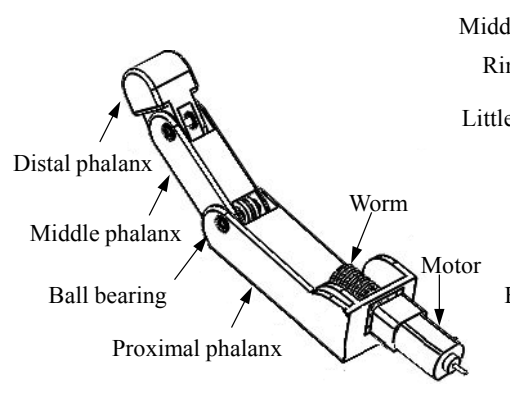

(a)

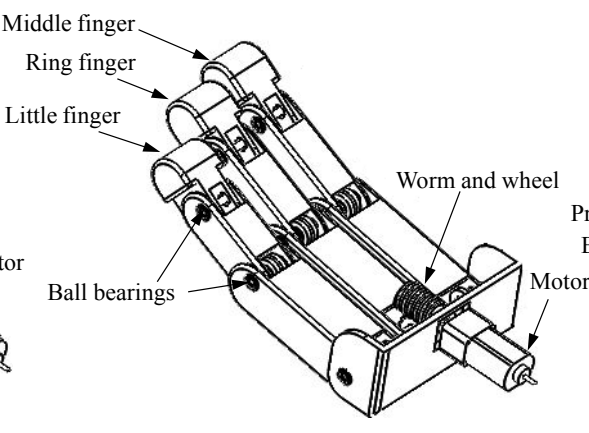

(b)

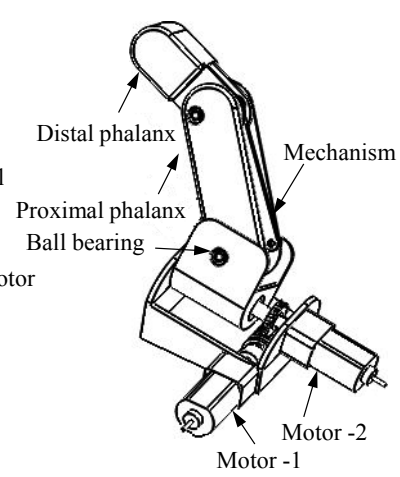

(c)

Figure 7. Finger units of the proposed hand prosthesis. (a) First finger unit. This consists of three phalanxes, worm and wheel, and a motor; (b) Second finger unit. Middle, ring and little fingers; and a motor are available in this unit; (c) Third finger unit. This includes thumb with proximal phalanx and distal phalanx, and 2 motors.

fingers, worm and wheel gears (reduction ratio 35:1), and a motor. These three fingers correspond to the middle, ring and little fingers of the human hand [refer Figure $7(\mathrm{~b})]$ and they are actuated together by a single motor using a single 
shaft as shown in Figure 7(b). Third finger unit consists of thumb, its worm and wheel gears (reduction ratio 35:1) and two DC motors [refer Figure 7(c)] which are perpendicular to each other. All four motors of the prototype of hand prosthesis have the same specification shown in Table 2. Finger structures, shafts and gears are fabricated from Al7075, stainless steel and Nylon 101 respectively using CNC machine.

All finger units are attached on the palm as shown in Figure 6. The proposed finger mechanism shown in Figure 1 is used to each finger in the first and second finger units. Motors of first and second finger units are connected to the MCP joint. Thumb can generate flexion/extension of the MCP and IP joints using motor -1 and opposition/apposition of thumb are generated using the motor -2 [refer Figure 6 and Figure $7(\mathrm{c})$ ]. The hand prosthesis assists user to generate cylindrical grasp, hook grasp, lateral pinch and tip pinch and palmar pinch shown in Figure 8.

\section{Experiment and Results}

Simulations and experiments are carried out to compare and verify the motion generation of the proposed finger. Furthermore, experiments are carried out to verify the adaptation ability of the finger and hand prosthesis. The kinematic model is simulated in MATLAB/Simulink environment to achieve the fingertip motion. Index finger motion of prosthesis is captured using a camera by placing passive markers to each joint and captured data is used to derive joint angles.

The experimental set-up is shown in the Figure 9. As the controller, ATmega 2560 (Atmel) is used. The selected motor driver is a dual H-bridge motor driver (L298N). PD control is applied in the joint space to generate the torque command for the MCP joint. As the desired motion MCP motion shown in Figure 11 which is generated from the simulation is used.

\section{Simulation and Experimental Results}

Figure 10 shows the trajectory of the tip of the index finger derived from simulation. Origin of the coordinate system $(0,0)$ is located at the center of the MCP joint. MCP angle variation of index finger is shown in Figure 11. Figure 12

Table 2. Specification of the motor.

\begin{tabular}{cc}
\hline Specification & Description \\
\hline Maximum Speed & $400 \mathrm{rpm}$ \\
Stall torque & $1.58 \mathrm{kgcm}$ \\
Gear ratio & $75: 1$ \\
Voltage & $6 \mathrm{~V}$ \\
No load current & $70 \mathrm{~mA}$ \\
Max. current & $1600 \mathrm{~mA}$ \\
Weight & $10 \mathrm{~g}$ \\
Encoder resolution & $20 \mathrm{cpr}$ \\
\hline
\end{tabular}




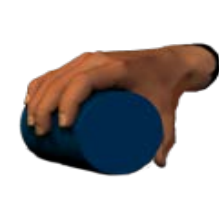

(a)

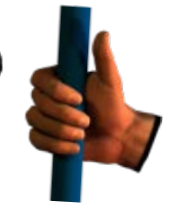

(b)

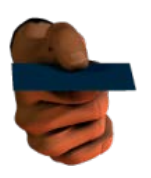

(c)

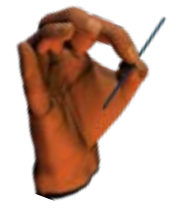

(d)

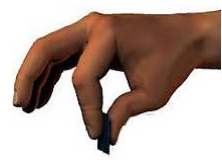

(e)

Figure 8. Achievable grasping of the hand prosthesis [18]. (a) Cylindrical grasp; (b) Hook grasp; (c) Lateral pinch; (d) Tip pinch; (e) Palmar pinch.

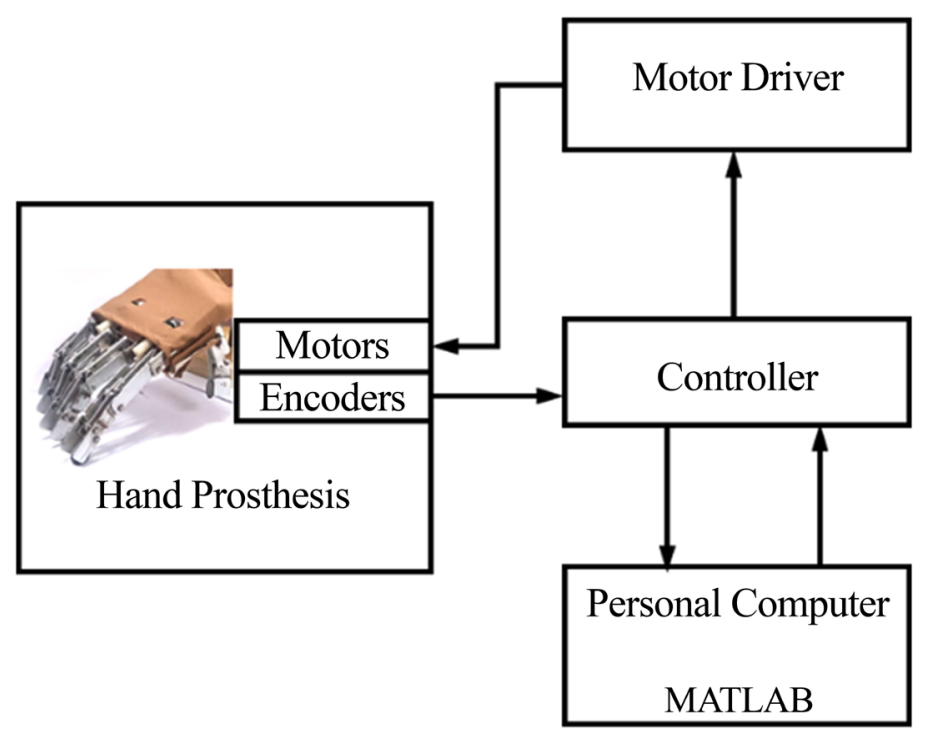

Figure 9. Experimental set-up.

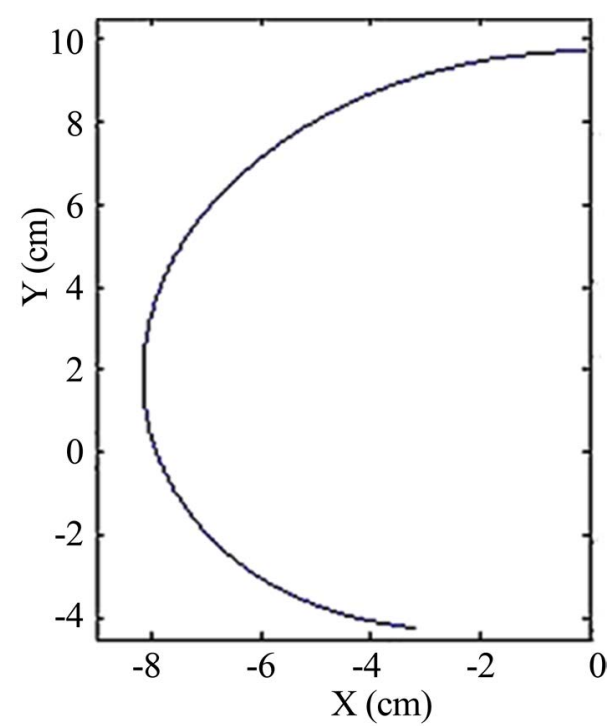

Figure 10. Simulation results of fingertip trajectory.

displays the angle of PIP with respective to the proximal phalanx. The angle of DIP with respect to the middle phalanx is shown in Figure 13. 


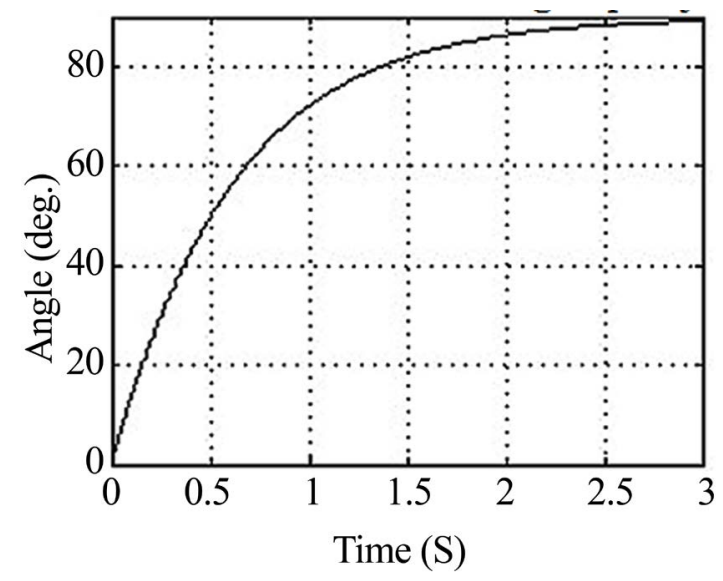

Figure 11. MCP angle of index finger.

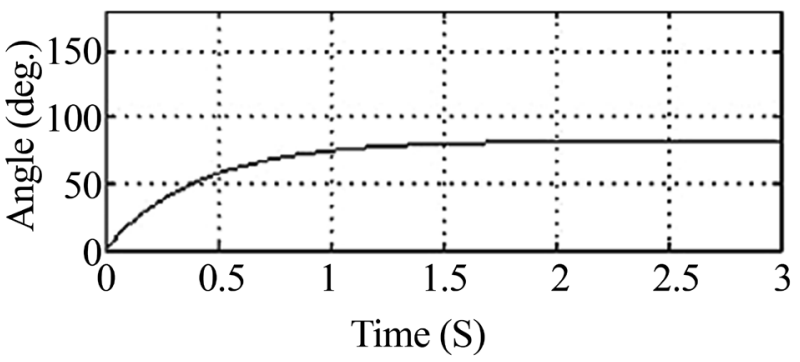

Figure 12. PIP angle of index finger.

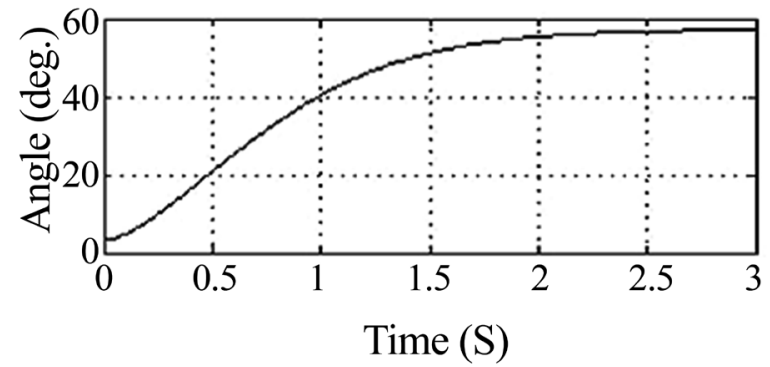

Figure 13. DIP angle of index finger.

Fingertip trajectory for human hand [26], MATLAB simulation and the experimental values are compared in Figure 14. Minor deviation of the simulation and experimental results are caused by frictional losses, dimensional tolerances associated to fabrication process, differences in spring constants and errors of motion capturing. The fingertip trajectory in Cartesian space for fabricated hand prosthesis is almost similar to actual hand.

Snapshots shown in Figure 15 and Figure 16 illustrate the adaptation ability of middle phalanx of index finger and distal phalanx of index finger respectively. Cylindrical grasping of the hand prosthesis is shown from the sequence of snapshots in Figure 17 and Figure 18 shows sequence of snapshots for hook grasp generation. Figure 15, Figure 16 and Figure 17 have verified the adaptation ability of the hand prosthesis. 


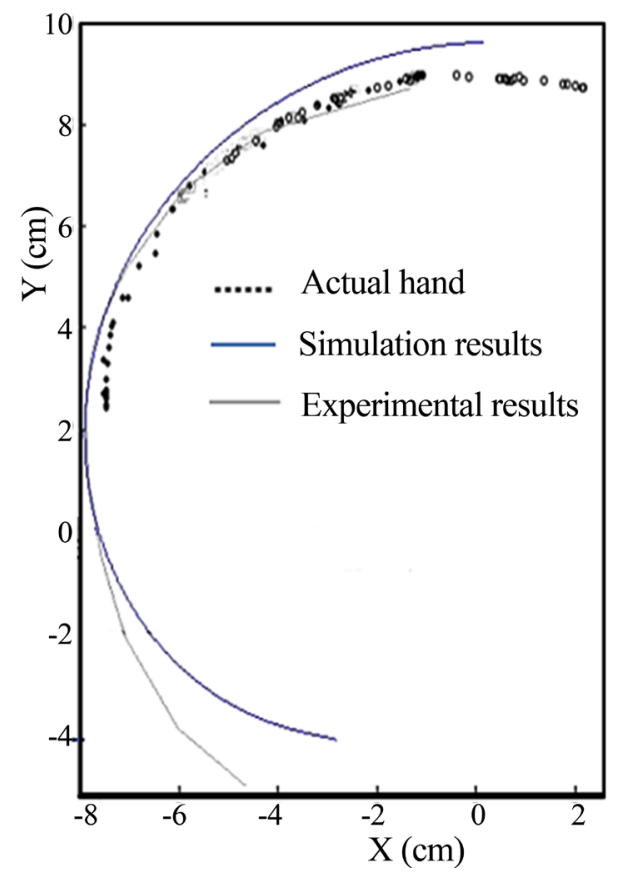

Figure 14. Comparison of finger-tip trajectory.
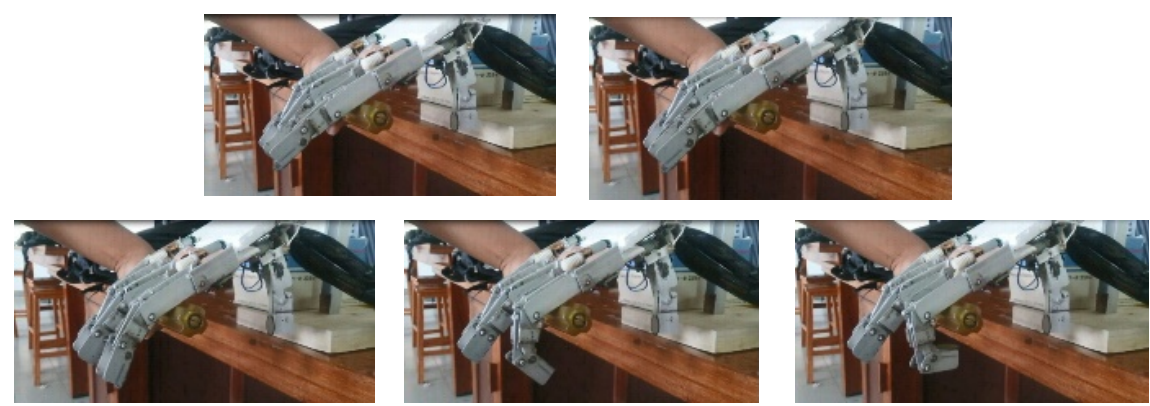

Figure 15. Sequence of images for adaptation of middle phalanx of index finger.
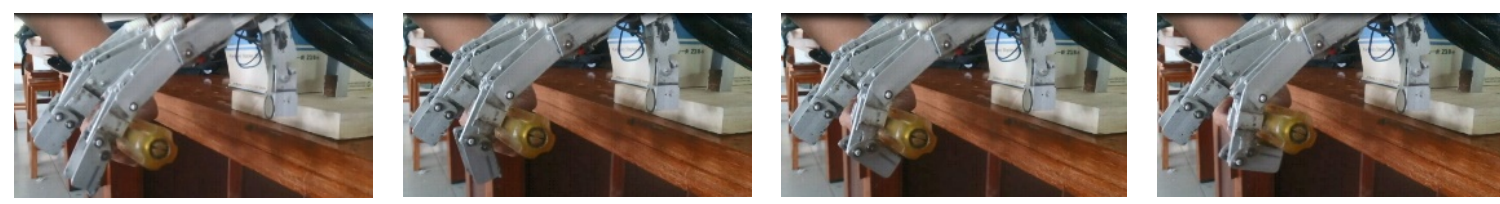

Figure 16. Sequence of images for adaptation of distal phalanx of index finger.

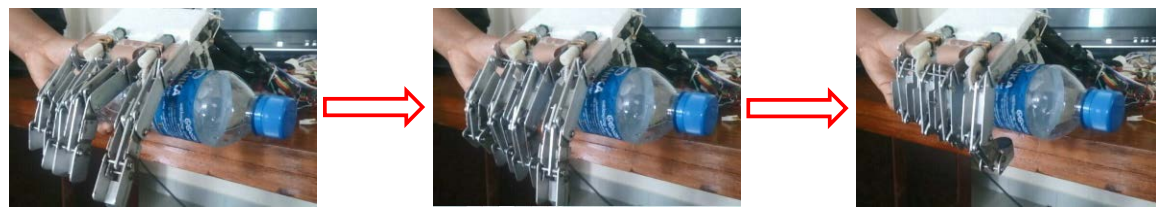

Figure 17. Snapshots of cylindrical grasping.

Table 3 shows motion ranges of MCP, PIP and DIP joints of the proposed index finger which are obtained from the simulation and experiments. Motion ranges of MCP, PIP and DIP joints of human index finger is also given in the 


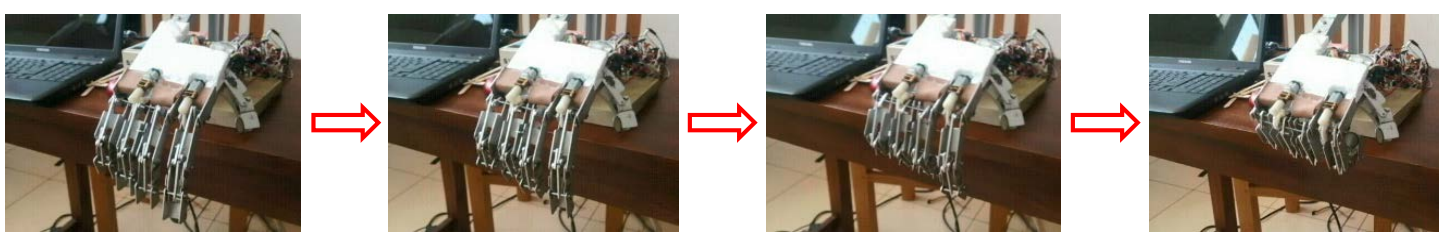

Figure 18. Snapshots of hook grasping.

Table 3. Movable ranges.

\begin{tabular}{cccc}
\hline & $\begin{array}{c}\text { MCP joint } \\
\text { [Degrees] }\end{array}$ & PIP joint [Degrees] & $\begin{array}{c}\text { DIP joint } \\
\text { [Degrees] }\end{array}$ \\
\hline $\begin{array}{c}\text { Human hand [17] } \\
\text { Simulation results } \\
\quad \text { (with 3D model) }\end{array}$ & $0-90$ & $0-110$ & $0-70$ \\
$\begin{array}{c}\text { Experiment results (with } \\
\text { fabricated hand prosthesis) }\end{array}$ & $0-90$ & $3-104$ & $0-82$ \\
\hline
\end{tabular}

table for the comparison. MCP joint of the hand prosthesis have the same movable range as the human hand. However, motion range of PIP and DIP joints has slight variation. The fabricated proposed hand prosthesis is unable to achieve the exact ranges of the 3D model. These slight deviations cause due to friction between links and joints, dimensional accuracy and tolerances associated to fabrication process and actual spring constant ratios are different from calculated values.

\section{Discussion and Conclusions}

Table 4 shows a comparison of under-actuation of the proposed prosthesis with the prosthesis available in the literature. DOF and number of actuators of the hand of each prosthesis are given for the comparison. It is evident from the table that the proposed prosthesis and Vanderbilt Multigrasp Hand [20] are with the highest DOF by utilising the minimum number of actuators.

An under-actuated and self-adaptive finger was proposed together with a hand prosthesis. The finger consisted of mainly two four-bar mechanisms. Modified mechanism of the finger was used as the thumb mechanism. Furthermore, a hand prosthesis with the proposed fingers and thumb was introduced in the paper. The finger was capable of generating different passive angles for a PIP joint and a DIP joint for each flexion angle of MCP joint. In addition, DIP joint was capable of generating different angles for the same angle of PIP joint. Thumb mechanism allowed for powered articulated thumb opposition/apposition. The weight of prototype hand prosthesis is about $250 \mathrm{~g}$. Kinematic analysis and computer simulations displayed that the finger mechanism was capable of performing required motions. Simulations were used to validate the movable ranges of the joint angles of finger. The movable ranges obtained from the experiments are $0^{\circ}-90^{\circ}, 5^{\circ}-90^{\circ}$ and $2^{\circ}-88^{\circ}$ for MCP, PIP and DIP respectively. Joint angle variation for MCP, PIP and DIP joints of the finger was obtained using simulations 
Table 4. Comparison of under-actuation.

\begin{tabular}{ccc}
\hline Prosthesis & DOF & No. of actuators \\
\hline Proposed Hand prosthesis & 9 & 4 \\
UOM Trans-radial Prosthesis [16] & 7 & 3 \\
DEKA RC Gen3 arm [19] & 6 & 4 \\
Vanderbilt Multigrasp Hand [20] & 9 & 4 \\
FluidHand III [21] & 8 & 5 \\
\hline
\end{tabular}

and experiments. The developed hand prosthesis offers a grasp adaptation using four actuators.

The hand prosthesis can be used to substitute a lost hand part of an amputee or can be used a terminal device for an arm prosthesis such as trans-radial prosthesis of trans-humeral prosthesis. Electromyography signals or electroencephalography signals of the wearer can be used to identify the motion intentions of the user to control the hand prosthesis, accordingly.

\section{Acknowledgements}

The authors would like to acknowledge the support given by Senate Research Council of University of Moratuwa, Sri Lanka (grant no: SRC/LT/2012/07).

\section{Conflicts of Interest}

The authors declare no conflicts of interest regarding the publication of this paper.

\section{References}

[1] Carrozza, M.C., Dario, P., Vecchi, F., Roccella, S., Zecca, M. and Sebastiani, F. (2003) The Cyberhand: on the Design of a Cybernetic Prosthetic Hand Intended to Be Interfaced to the Peripheral Nervous System. IEEE/RSJ International Conference on Intelligent Robots and Systems, Las Vegas, 27-31 October 2003, 2642-2647. https://doi.org/10.1109/IROS.2003.1249269

[2] Light, C.M. and Chappell, P.H. (2000) Development of a Lightweight and Adaptable Multiple-Axis Hand Prosthesis. Medical Engineering \& Physics, 22, 679-684. https://doi.org/10.1016/S1350-4533(01)00017-0

[3] Massa, B., Roccella, S., Carrozza, M.C. and Dario, P. (2002) Design and Development of an Underactuated Prosthetic Hand. IEEE International Conference on Robotics and Automation, Washington DC, 11-15 May 2002, 3374-3379. https://doi.org/10.1109/ROBOT.2002.1014232

[4] Cutkosky, M.R. (1989) On Grasp Choice, Grasp Models, and the Design of Hands for Manufacturing Tasks. IEEE Transactions on Robotics and Automation, 5, 269-279. https://doi.org/10.1109/70.34763

[5] Bekey, G.A., Tomovic, R. and Zeljkovic, I. (1990) Control Architecture for the Belgrade/USC Hand. In: Venkataraman, S.T. and Iberall, T., Eds., Dextrous Robot Hands, Springer, New York, 136-149. https://doi.org/10.1007/978-1-4613-8974-3_7

[6] Salisbury, J.K. and Craig, J.J. (1982) Articulated Hands Force Control and Kinemat- 
ic Issues. International Journal of Robotics Research, 1, 4-17. https://doi.org/10.1177/027836498200100102

[7] Cipriani, C., Controzzi, M. and Carrozza, M.C. (2011) The Smart Hand Transradial Prosthesis. Journal of Neuroengineering and Rehabilitation, 8, 29. https://doi.org/10.1109/IROS.2009.5353919

[8] Laliberte, T., Birglen, L. and Gosselin, C. (2002) Underactuation in Robotic Grasping Hands. Mach. Intell. Robot. Control, 4, 1-11.

[9] Cabas, R., Cabas, L.M. and Balaguer, C. (2006) Optimized Design of the Underactuated Robotic Hand. IEEE International Conference on Robotics and Automation, Orlando, 15-19 May 2006, 982-987. https://doi.org/10.1109/ROBOT.2006.1641837

[10] Carrozza, M.C., Suppo, C., Sebastiani, F., Massa, B., Vecchi, F., Lazzarini, R., Cutkosky, M.R. and Dario, P. (2004) The SPRING Hand: Development of a Self-Adaptive Prosthesis for Restoring Natural Grasping. Autonomous Robots, 16, 125-141. https://doi.org/10.1023/B:AURO.0000016863.48502.98

[11] Gosselin, C., Pelletier, F. and Laliberte, T. (2008) An Anthropomorphic Underactuated Robotic Hand with 15 Dofs and a Single Actuator. IEEE International Conference on Robotics and Automation, Pasadena, 19-23 May 2008, 749-754. https://doi.org/10.1109/ROBOT.2008.4543295

[12] Krut, S. (2005) A Force-Isotropic Underactuated Finger. IEEE International Conference on Robotics and Automation, Barcelona, 18-22 April 2005, 2314-2319. https://doi.org/10.1109/ROBOT.2005.1570458

[13] Wu, L., Carbone, G. and Ceccarelli, M. (2009) Designing an Underactuated Mechanism for a 1 Active DOF Finger Operation. Mechanism and Machine Theory, 44, 336-348. https://doi.org/10.1016/j.mechmachtheory.2008.03.011

[14] Bandara, D.S.V., Gopura, R.A.R.C., Kajanthan, G., Brunthavan, M. and Abeynayake, H.I.M.M. (2014) An Under-Actuated Mechanism for a Robotic Finger. IEEE International Conference on Cyber Technology in Automation, Control, and Intelligent Systems, Hong Kong, 4-7 June 2014, 407-412.

https://doi.org/10.1109/CYBER.2014.6917498

[15] Zhao, D.W., Jiang, L., Huang, H., Jin, M.H., Cai, H.G. and Liu, H. (2006) Development of a Multi-DOF Anthropomorphic Prosthetic Hand. IEEE International Conference on Robotics and Biomimetics, Kunming, 17-20 December 2006, 878-883. https://doi.org/10.1109/ROBIO.2006.340336

[16] Bandara, D.S.V., Gopura, R.A.R.C., Hemapala, K.T.M.U. and Kiguchi, K. (2014) A Multi-DoF Anthropomorphic Transradial Prosthetic Arm. IEEE RAS/EMBS International Conference on Biomedical Robotics and Biomechatronics, Sao Paulo, 12-15 August 2014, 1039-1044. https://doi.org/10.1109/BIOROB.2014.6913917

[17] Love, L.J., Lind, R.F. and Jansen, J.F. (2009) Mesofluidic Actuation for Articulated Finger and Hand Prosthetics. IEEE/RSJ International Conference on Intelligent Robots and Systems, St. Louis, 10-15 October 2009, 2586-2591.

[18] Feix, T., Romero, J., Schmiedmayer, H., Dollar, A. and Kragic, D. (2016) The GRASP Taxonomy of Human Grasp Types. IEEE Transactions on Human-Machine Systems, 46, 66-77. https://doi.org/10.1109/THMS.2015.2470657

[19] Resnik, L., Klinger, S. and Etter, K. (2013) The DEKA Arm: Its Features, Functionality, and Evolution during the Veterans Affairs Study to Optimize the DEKA Arm. Prosthetics and Orthotics International, 38, 492-504. https://doi.org/10.1177/0309364613506913

[20] Dalley, S., Bennett, D. and Goldfarb, M. (2014) Functional Assessment of the Vanderbilt Multi Grasp Myoelectric Hand: A Continuing Case Study. International 
Conference of the IEEE Engineering in Medicine and Biology Society, Chicago, 26-30 August 2014, 6195-6198. https://doi.org/10.1109/EMBC.2014.6945044

[21] Gaiser, I.N., Pylatiuk, C., Schulz, S., Kargov, A., Oberle, R. and Werner, T. (2009) The FLUIDHAND III: A Multifunctional Prosthetic Hand. Journal of Prosthetics and Orthotics, 21, 91-96. https://doi.org/10.1097/JPO.0b013e3181a1ca54

[22] Wang, X., Zhao, J., Yang, D., Li, N., Sun, C. and Liu, H. (2010) Biomechatronic Approach to a Multi-Fingered Hand Prosthesis. IEEE RAS \& EMBS International Conference on Biomedical Robotics and Biomechatronics, Tokyo, 26-29 September 2010, 209-214. https://doi.org/10.1109/BIOROB.2010.5627734

[23] Ceccarelli, M. and Zottola, M. (2017) Design and Simulation of an Underactuated Finger Mechanism for LARM Hand. Robotica, 35, 483-497.

https://doi.org/10.1017/S0263574715000648

[24] Controzzi, M., Clemente, F., Barone, D., Ghionzoli, A. and Cipriani, C. (2017) The SSSA-MyHand: A Dexterous Lightweight Myoelectric Hand Prosthesis. IEEE Transactions on Neural Systems and Rehabilitation Engineering, 25, 459-468. https://doi.org/10.1109/TNSRE.2016.2578980

[25] Gopura, R.A.R.C., Bandara, D.S.V., Gunasekera, N.P.A., Hapuarachchi, V.H. and Ariyarathna, B.S. (2017) A Prosthetic Hand with Self-Adaptive Fingers. IEEE International Conference on Control, Automation and Robotics, Nagoya, 24-26 April 2017, 269-274. https://doi.org/10.1109/ICCAR.2017.7942701

[26] Kamper, D.G., Cruz, E.G. and Siegel, M.P. (2003) Stereotypical Fingertip Trajectories during Grasp. Journal of Neurophysiology, 90, 3702-3710.

https://doi.org/10.1152/jn.00546.2003 